\title{
BIBECHANA
}

A Multidisciplinary Journal of Science, Technology and Mathematics

ISSN 2091-0762 (online)

Journal homepage: http://nepjol.info/index.php/BIBECHANA

\section{Periodic Solution of the restricted three body problem}

\author{
M.R. Hassan ${ }^{1}$, R. R.Thapa ${ }^{2 *}$ \\ ${ }^{1}$ Dept. of Mathematics, S.M. College, Bhagalpur, Under T.M.B.University, Bhagalpur \\ ${ }^{2}$ Dept. of Mathematics, P.G.Campus, Biratnagar, Morang, Nepal \\ *Email: thaparajuram@yahoo.com
}

Article history: Received 25 August, 2013; Accepted 5 October, 2013

\section{Abstract}

The effect of perturbation in centrifugal force on the periodic solution of the restricted three-body problem representing analytic continuation of Keplerian rectilinear periodic motion has been examined. However, we have taken the perturbation in the centrifugal force to be of the order of $\mu$, the reduced mass of the smaller primary. We have calculated the first order perturbations also.

Keywords: Elliptic restricted three body problem; Generating solution; Hamiltonian equations; Perturbation; Periodic solution; Variational equations.

\section{Introduction}

Considering the two body problem, Poincare [1] classified three kinds of periodic solutions of the restricted three body problem. Solutions of I and II kinds are related to the planar case of the three body problem. In the solution of I kind, the eccentricity reduces to zero together with small mass. In the solution of II kind, the eccentricity does not reduce to zero with the small mass. Poincare studied in details the I kind and proved its existence. For the solution of the II kind, he deduced conditions under which they may exist but regorious proof for the existence of the solution of this kind was not given by him. Poincare used the De-Launay elements in his study and so he excluded the case when one of the eccentricity of the generating solution is equal to unity. Kurcheeva [2] considered the last case. Under certain conditions she proved the existence of so called periodic solution of Poincare i.e. having equal periods for their perturbed as well as for the unperturbed motions.

Ahmad [3] studied the elliptic restricted three body problem generalizing the work of Kurcheeva. Ahmad and Khan [4] generalized the work of Ahmad with the introduction of perturbation in the Coriolis force.

In this paper we have studied the generalization of the restricted three body problem introducing perturbation in the centrifugal force. We have examined the existence of the periodic solution taking the perturbation to be of the order of $\mu$, the reduced mass of the smaller primary. We have also calculated first order perturbation.

\section{Hamiltonian Equations of Motion}

Using non-dimensional variables and a synodic system of co-ordinates, the Hamiltonian equations of motion are 
where

$$
\begin{aligned}
& \frac{\mathrm{dX}}{\mathrm{dt}}=\frac{\partial \mathrm{H}}{\partial \mathrm{X}}, \frac{\mathrm{dy}}{\mathrm{dt}}=\frac{\partial \mathrm{H}}{\partial \mathrm{Y}} \\
& \frac{\mathrm{dX}}{\mathrm{dt}}=-\frac{\partial \mathrm{H}}{\partial \mathrm{x}}, \frac{\mathrm{dY}}{\mathrm{dt}}=-\frac{\partial \mathrm{H}}{\partial \mathrm{y}}
\end{aligned}
$$

$$
\begin{aligned}
& \mathrm{H}=\frac{1}{2}\left(\mathrm{X}^{2}+\mathrm{Y}^{2}\right)+(\mathrm{yX}+\mathrm{xY})-\frac{1-\mu}{\mathrm{r}_{1}}-\frac{\mu}{\mathrm{r}_{2}} \\
& \mathrm{r}_{1}^{2}=(\mathrm{x}-\mu)^{2}+\mathrm{y}^{2} \\
& \mathrm{r}_{2}^{2}=(\mathrm{x}-\mu+1)^{2}+\mathrm{y}^{2}
\end{aligned}
$$

In the above equations, the parameter $\mu$ is the ratio of the mass of the smaller primary to the total mass of the primaries and $0<\mu \leq 1 / 2$.

We consider the perturbation in the centrifugal force with the help of the parameter $\beta$, the unperturbed value of $\beta$ being unity. The corresponding Hamiltonian function takes the following Bhatnagar and Hallan form [5]

$$
\mathrm{H}=\frac{1}{2}\left(\mathrm{X}^{2}+\mathrm{Y}^{2}\right)+(\mathrm{Xy}+\mathrm{Yx})+\frac{1}{2}(1-\beta)\left(\mathrm{x}^{2}+\mathrm{y}^{2}\right)-\frac{1-\mu}{\mathrm{r}_{1}}-\frac{\mu}{\mathrm{r}_{2}}
$$

Here $\beta$ may be taken as

$$
\beta=1+\epsilon^{\prime},\left|\in{ }^{\prime}\right|<<1
$$

where $\in$ ' represents the perturbation in the centrifugal force. Upto first order, we have

$$
\mathrm{H}=\frac{1}{2}\left(\mathrm{X}^{2}+\mathrm{Y}^{2}\right)+(\mathrm{Xy}-\mathrm{Yx})-\frac{\epsilon^{\prime}}{2}\left(\mathrm{x}^{2}+\mathrm{y}^{2}\right)-\frac{1-\mu}{\mathrm{r}_{1}}-\frac{\mu}{\mathrm{r}_{2}}
$$

\section{Limiting case $\mu=0$}

For the elimination of singularity at the bigger primary we shall use Levi-Civita's [6] variables as used by Kurcheeva:

$$
\begin{aligned}
& \mathrm{x}+\mu+\mathrm{iy}=(\mathrm{p}+\mathrm{iq})^{2} \\
& X-\mathrm{i}(\mathrm{Y}+\mu)=\frac{\mathrm{P}-\mathrm{iQ}}{2(\mathrm{P}+\mathrm{iq})} \\
& \frac{\mathrm{dt}}{\mathrm{ds}}=4\left(\mathrm{p}^{2}+\mathrm{q}^{2}\right)
\end{aligned}
$$

The regularized canonical equations of motion became

$$
\begin{aligned}
& \frac{\mathrm{dp}}{\mathrm{dS}}=\frac{\partial \Omega}{\partial \mathrm{P}}, \frac{\mathrm{dq}}{\mathrm{dS}}=\frac{\partial \Omega}{\partial \mathrm{Q}} \\
& \frac{\mathrm{dP}}{\mathrm{d} S}=\frac{\partial \Omega}{\partial \mathrm{p}}, \frac{\mathrm{dQ}}{\mathrm{dS}}=\frac{\partial \Omega}{\partial \mathrm{q}}
\end{aligned}
$$

where

$$
\begin{aligned}
& \Omega=\frac{1}{2}\left(\mathrm{P}^{2}+\mathrm{Q}^{2}\right)+2 \mathrm{r}_{1}(\mathrm{qP}-\mathrm{pQ})+4 \mu\left(\mathrm{p}^{4}-\mathrm{q}^{4}\right)-4+4 \mu-4 \mu\left(\mathrm{r}_{1} / \mathrm{r}_{2}\right) \\
& +2 \mathrm{Cr}_{1}-2 \in \in^{\prime} \mathrm{r}_{1}^{3}-4 \mu \in \in^{\prime}\left(\mathrm{p}^{4}-\mathrm{q}^{4}\right)
\end{aligned}
$$




$$
r_{1}=p^{2}+q^{2}, r_{2}^{2}=1-2\left(p^{2}+q^{2}\right)+\left(p^{2}+q^{2}\right)^{2}
$$

The solution of the system (7) being the solution of the system (8), we must have

$$
\Omega=0
$$

We suppose that $\epsilon^{\prime}$ is or the order of $\mu$ i.e. $\epsilon^{\prime}=\epsilon_{0}^{\prime} \mu$. If we put $\mu=0$ in the system (7) may be integrated. The solutions of the simplified system, which in sideral system of co-ordinates corresponds to collinear motion, has Krasinski form [7].

$$
\begin{aligned}
& \mathrm{p}_{\mathrm{O}}=\rho \sin \theta, \mathrm{q}_{\mathrm{O}}=\rho \cos \theta \\
& \mathrm{P}_{\mathrm{O}}=\dot{\rho} \sin \theta, \mathrm{Q}_{\mathrm{O}}=\dot{\rho} \cos \theta
\end{aligned}
$$

where

$$
\begin{aligned}
& \rho=\sqrt{2} / \mathrm{C} \cos \left(2 \sqrt{\mathrm{C}} \mathrm{S}-\phi_{0}\right) \\
& \theta=\frac{1}{2 \mathrm{n}}\left[4 \sqrt{\mathrm{C}} \mathrm{S}+\sin \left(4 \sqrt{\mathrm{C}} \mathrm{S}-2 \phi_{0}\right)\right] \\
& \mathrm{n}=\left(\sqrt{\mathrm{C}_{0}}\right)^{3}=\text { mean motion, } \dot{\rho}=\frac{\mathrm{d} \rho}{\mathrm{dS}}
\end{aligned}
$$

The period of such a solution is

$$
\mathrm{S}=\left\{\begin{array}{l}
\frac{\pi \mathrm{K}}{2 \sqrt{\mathrm{C}}_{0}} \text { if }(\mathrm{k}+\mathrm{m}) \text { is an even number } \\
\frac{\pi \mathrm{K}}{\sqrt{\mathrm{C}}_{0}} \text { if }(\mathrm{k}+\mathrm{m}) \text { is an odd number }
\end{array}\right.
$$

The relation between $\mathrm{t}$ and $\mathrm{s}$ for $\mu=0$ is given by

$$
\mathrm{t}_{0}=2(\theta-\omega)=\frac{1}{\mathrm{n}}\left[4 \sqrt{\mathrm{C}} \mathrm{S}+\sin \left(4 \sqrt{\mathrm{C}} \mathrm{S}-2 \phi_{0}\right)\right]
$$

\section{Solutions of the Variational Equations of the Generating System}

The solutions of the variational equations of the system (7) for $\mu=0$ are well known. The solutions given by Krasinski are

$$
\begin{aligned}
& \delta \mathrm{p}_{1}=\mathrm{q}_{0}, \delta \mathrm{p}_{2}=\dot{\mathrm{p}}_{0}, \delta \mathrm{p}_{3}=\mathrm{p}_{0}+\mathrm{tq}_{0}, \delta \mathrm{p}_{4}=\left(-\frac{1}{2 \sqrt{\mathrm{C}_{0}}} \mathrm{Q}_{0}+\frac{2}{\mathrm{n}} \dot{\mathrm{p}}_{0} \mathrm{~S}-\frac{\mathrm{t}_{0} \mathrm{q}_{0}}{\mathrm{n}}\right) \\
& \delta \mathrm{q}_{1}=-\mathrm{p}_{0}, \delta \mathrm{q}_{2}=\dot{\mathrm{q}}_{0}, \delta \mathrm{q}_{3}=\mathrm{q}_{0}-\mathrm{t}_{0} \mathrm{p}_{0}, \delta \mathrm{q}_{4}=\left(\frac{1}{2 \sqrt{\mathrm{C}_{0}}} \mathrm{P}_{0}+\frac{2}{\mathrm{n}} \dot{\mathrm{q}}_{0} \mathrm{~S}-\frac{\mathrm{t}_{0} \mathrm{p}_{0}}{\mathrm{n}}\right) \\
& \delta \mathrm{P}_{1}=\mathrm{Q}_{0}, \delta \mathrm{P}_{2}=\dot{\mathrm{P}}_{0}, \delta \mathrm{P}_{3}=\mathrm{P}_{0}+\mathrm{t}_{0} \mathrm{Q}_{0}, \delta \mathrm{p}_{4}=\left(2 \sqrt{\mathrm{C}_{0}} \mathrm{q}_{0}+\frac{2}{\mathrm{n}} \dot{\mathrm{p}}_{0} \mathrm{~S}+\frac{2}{\mathrm{n}} \mathrm{p}_{0}-\frac{\mathrm{t}_{0} \mathrm{Q}_{0}}{\mathrm{n}}\right) \\
& \delta \mathrm{Q}_{1}=-\mathrm{P}_{0}, \delta \mathrm{Q}_{2}=\dot{\mathrm{Q}}_{0}, \delta \mathrm{Q}_{3}=\mathrm{Q}_{0}-\mathrm{t}_{0} \mathrm{P}_{0}, \delta \mathrm{Q}_{4}=\left(-2 \sqrt{\mathrm{C}_{0}} \mathrm{p}_{0}+\frac{2}{\mathrm{n}} \dot{\mathrm{Q}}_{0} \mathrm{~S}+\frac{2}{\mathrm{n}} \mathrm{Q}_{0}+\frac{\mathrm{t}_{0} \mathrm{P}_{0}}{\mathrm{n}}\right)
\end{aligned}
$$

\section{Proof of Isoperiodic Solution}

We see that our Hamiltonian function (8) possesses the same properties as that of Kurcheeva. Therefore, under the conditions: 
and $\quad \begin{aligned} \mathrm{q}(\mathrm{o}) & =\mathrm{p}(\mathrm{o})=\mathrm{o}, \mathrm{p}\left(\mathrm{S}^{*}\right)=\mathrm{Q}\left(\mathrm{S}^{*}\right)=0, \mathrm{~S}^{*}=\mathrm{S} / 4 \\ \mathrm{q}(\mathrm{o}) & =\mathrm{p}(\mathrm{o})=\mathrm{o}, \mathrm{q}\left(\mathrm{S}^{*}\right)=\mathrm{P}\left(\mathrm{S}^{*}\right)=0, \mathrm{~S}^{*}=\mathrm{S} / 2\end{aligned}$

the general solutions $x_{i}(s),(i=1,2,3,4)$ are periodic with period $S$ as in Kurcheeva where by $x_{i}(i=$ $1,2,3,4), \mathrm{p}, \mathrm{q}, \mathrm{P}, \mathrm{Q}$ are respectively denoted.

The solutions (10) of the generating system satisfy the conditions (14) when $\mathrm{k}+\mathrm{m}$ is odd and (15) when $\mathrm{k}+\mathrm{m}$ is even under the following values of the parameters $\phi_{0}$ and $\omega$.

$$
\begin{aligned}
& \text { I. } \phi_{0}=0, \omega= \pm \pi / 2 \\
& \text { II. } \phi_{0}=0= \pm \pi / 2, \omega=\pi \mathrm{L}, \mathrm{L}=0,1,2, \ldots \ldots \text {. }
\end{aligned}
$$

We observe that the value of $\Omega_{0}$ is the same as in Kurcheeva.

Hence one can follow the work of Kurcheeva, step by step, to examine the existence of isoperiodic solutions. The isoperiodic solution exist for $\phi_{0}=0, \omega= \pm \pi / 2, \mathrm{k}$ - even, $\mathrm{m}$ - odd.

For arbitrary $\mathrm{k}$ and $\mathrm{m}$, there exist periodic solutions reducing to the generating one for $\mu=0$. The period of these solutions is an analytic function of $\mu, S^{*} S^{*}+4 S(\mu), S^{*}$ being the period of the generating solution.

\section{Perturbation of the First Order}

In the proceeding paragraph the existence of the periodic orbits analytic relative to $\mu$ with the period $\mathrm{S}=\mathrm{S}^{*}+\delta \mathrm{S}$, where $\mathrm{S}^{*}$ is the period of the generating solution has been pointed out. Let us pass on to such time $t$ the period in which does not depend on $\mu$ :

$$
\tau=\frac{\mathrm{S}^{*}}{\mathrm{~S} *+\delta \mathrm{S}}=\frac{\mathrm{S} *}{\mathrm{~S} *+\alpha} \mathrm{S}
$$

Where $\mathrm{S}^{*}=\frac{\pi \mathrm{k}}{4 \sqrt{\mathrm{C}}}$. Then the equations of motion are written as

$$
\frac{\mathrm{dx}_{\mathrm{i}}}{\mathrm{d} \tau}=\frac{\mathrm{S}^{*}+\alpha}{\mathrm{S} *} \mathrm{X}_{\mathrm{i}}\left(\mathrm{x}_{1}, \ldots \ldots \ldots \mathrm{x}_{4}, \mathrm{C}\right),(\mathrm{i}=1, \ldots \ldots \ldots, 4)
$$

The solution of the system (16) may be sought in the form of series in integral positive powers of $\mu$.

$$
\mathrm{x}_{\mathrm{k}}=\mathrm{x}_{\mathrm{k}}^{(0)}+\sum_{\mathrm{i}=1}^{\infty} \mathrm{x}_{\mathrm{k}}^{(\mathrm{i})} \mu^{\mathrm{i}},(\mathrm{k}=1, \ldots .4)
$$

For $\mathrm{C}$ and $\alpha$, we have

$$
\mathrm{C}=\mathrm{C}_{0}+\sum_{\mathrm{i}=1}^{\infty} \mathrm{C}_{\mathrm{i}} \mu^{\mathrm{i}}, \alpha=\sum_{\mathrm{i}=1}^{\infty} \alpha_{\mathrm{i}} \mu^{\mathrm{i}}
$$

As it is proved by Kurcheeva series (17) and (18) representing solutions of the system (16) converge for sufficiently small value of $\mu$.

Let us substitute (17), (18) in (16) for the determination of the functions $x_{i}^{(j)}(\tau)$. We have a system of linear differential equations.

$$
\frac{\mathrm{dx}_{\mathrm{k}}^{(\mathrm{i})}}{\mathrm{d} \tau}=\sum_{\mathrm{j}=1}^{4} \mathrm{p}_{\mathrm{kj}} \mathrm{x}_{\mathrm{j}}^{(\mathrm{i})}+\mathrm{x}_{\mathrm{k}}^{(0)} \mathrm{h}_{\mathrm{i}}+\phi_{\mathrm{k}} \mathrm{C}_{\mathrm{i}}+\mathrm{F}_{\mathrm{k}}^{(\mathrm{i})}
$$

where by $h_{i}$ we denote $\alpha_{i} / S^{*}$ 


$$
\begin{aligned}
& \mathrm{P}_{\mathrm{kj}}=\frac{\partial \mathrm{x}_{\mathrm{k}}^{(0)}}{\partial \mathrm{x}_{\mathrm{j}}}, \mathrm{x}_{\mathrm{k}}^{(0)}=\mathrm{x}_{\mathrm{k}}\left(\mathrm{x}_{1}^{(0)}, \ldots \ldots . \mathrm{x}_{4}^{(0)}, \mathrm{C}_{0}, 0\right) \\
& \phi_{\mathrm{k}}=\frac{\partial \mathrm{x}_{\mathrm{k}}}{\partial \mathrm{C}_{0}} \\
& \mathrm{~F}_{\mathrm{K}}^{(\mathrm{i})}=\mathrm{F}_{\mathrm{K}}^{(\mathrm{i})}\left(\mathrm{x}_{\mathrm{i}}^{(0)}, \ldots \ldots ., \mathrm{x}_{4}{ }^{(\mathrm{i}-1)}, \mathrm{C}_{0}, \ldots \ldots, \mathrm{C}_{\mathrm{i}-1}, \mathrm{~h}_{1}, \ldots \ldots, \mathrm{h}_{\mathrm{i}-1}\right)
\end{aligned}
$$

In particular for $\mathrm{i}=1$, we have

$$
\begin{aligned}
& \mathrm{F}_{1}^{(\mathrm{i})}=0 \\
& \mathrm{~F}_{2}^{(\mathrm{i})}=0 \\
& \mathrm{~F}_{3}^{(\mathrm{i})}=-16 \mathrm{q}^{3}+\frac{8 \mathrm{q}}{\mathrm{r}_{2}}+\frac{8 \mathrm{qr}_{1}\left(1+\mathrm{r}_{1}\right)}{\mathrm{r}_{2}^{3}}+12 \in_{0}^{\prime} \mathrm{r}_{1} \mathrm{p} . \\
& \mathrm{F}_{4}^{(\mathrm{i})}=16 \mathrm{p}^{3}+\frac{8 \mathrm{p}}{\mathrm{r}_{2}}-\frac{8 \mathrm{pr}_{1}\left(1-\mathrm{r}_{1}\right)}{\mathrm{r}_{2}^{3}}+12 \in_{0}^{\prime} \mathrm{r}_{1}^{2} \mathrm{q} . \\
& \phi_{1}=\phi_{2}=0, \phi_{3}=-4 \mathrm{p}_{0}, \phi_{4}=-4 \mathrm{q}_{0} \\
& \mathrm{x}_{1}^{(0)}=\mathrm{P}_{0}+2 \mathrm{rq}_{0}, \mathrm{x}_{2}^{(0)}=\mathrm{Q}_{0}-2 \mathrm{rp}_{0}, \mathrm{x}_{3}^{(0)}=2 \mathrm{rQ}_{0}-4 \mathrm{p}_{0} \mathrm{C}_{0}, \mathrm{x}_{4}=-2 \mathrm{rP}_{0}-4 \mathrm{q}_{0} \mathrm{C}_{0}
\end{aligned}
$$

All the linearly independent solutions of the system

$$
\frac{\mathrm{dy}_{\mathrm{i}}}{\mathrm{d} \tau}=\sum_{\mathrm{k}=1}^{4} \mathrm{p}_{\mathrm{ik}} \cdot \mathrm{y}_{\mathrm{k}}
$$

are obtained from the expression (13).

Let us seek particular solution of the system (19) with the help of the method of variation of arbitrary constant.

$$
\text { Let } \quad \mathrm{x}_{\mathrm{j}}^{(\mathrm{i})}=\sum_{\mathrm{k}=1}^{4} \mathrm{~L}_{\mathrm{k}} \cdot \mathrm{y}_{\mathrm{ik}}
$$

where $\left\{\mathrm{y}_{\mathrm{jk}}\right\}$ is a fundamental system of solution of the homogenous system

Differentiating (24), we obtain

$$
\sum_{\mathrm{k}=1}^{4} \mathrm{y}_{\mathrm{jk}} \frac{\mathrm{dL}}{\mathrm{d} \tau}=\phi_{\mathrm{j}} \mathrm{c}_{1}+\mathrm{x}_{\mathrm{j}}^{(\mathrm{i})} \mathrm{h}_{\mathrm{i}}+\mathrm{F}_{\mathrm{j}}^{(\mathrm{i})}
$$

Now, in order to get rid of $y_{j k}$ from the coefficients with $L_{k}$ depending on the time $\tau$. We shall multiply the expression (25) by $Z_{\mathrm{ji}}$, where $\left\{Z_{\mathrm{ji}}\right\}$ is a matrix of solution for conjugate system (23) we shall add then in $\mathrm{j}$ from 1 to 4 .

We have

$$
\sum_{\mathrm{k}=1}^{4} \mathrm{~A}_{\mathrm{ik}} \dot{\mathrm{L}}_{\mathrm{k}}=\mathrm{f}_{\mathrm{i}}
$$

where $\mathrm{A}_{\mathrm{ik}}=\sum_{\mathrm{j}=1}^{4} \mathrm{Z}_{\mathrm{ji}} \mathrm{y}_{\mathrm{jk}}$, do not depend on time (the property of the conjugate system)

$$
\mathrm{f}_{\mathrm{i}}=\sum_{\mathrm{k}=1}^{4}\left(\mathrm{x}_{\mathrm{k}}^{(\mathrm{o})} \mathrm{h}_{1}+\phi_{\mathrm{k}} \mathrm{C}_{1}+\mathrm{x}_{\mathrm{k}}^{(\mathrm{i})}\right) \mathrm{Z}_{\mathrm{ki}}
$$

As the expression (23) is a system in variation of a cononic sytem, so for the determination of $Z_{\mathrm{ij}}$ we have the following relations

$$
\mathrm{Z}_{1 \mathrm{i}}=-\mathrm{y}_{3 \mathrm{i}}, \mathrm{Z}_{2 \mathrm{i}}=-\mathrm{y}_{4 \mathrm{i}}, \mathrm{Z}_{3 \mathrm{i}}=-\mathrm{y}_{1 \mathrm{i}}, \mathrm{Z}_{4 \mathrm{i}}=-\mathrm{y}_{2 \mathrm{i}}
$$


As the coefficients Aij do not depend on time, so for the determination we shall put $\tau=0$. Using the formula (13) for the calculation of Yij (0), we find

$$
\left[\mathrm{A}_{\mathrm{ij}}\right]=\left[\begin{array}{cccc}
0 & 0 & 0 & \frac{4}{\sqrt{\mathrm{C}}} \\
0 & 0 & 8 & \frac{16}{\mathrm{n}} \\
0 & -8 & 0 & 0 \\
\frac{-4}{\sqrt{\mathrm{C}}} & \frac{-16}{\mathrm{n}} & 0 & 0
\end{array}\right]
$$

Thus we have the following system

$$
\begin{aligned}
& \dot{\mathrm{L}}_{4}=\frac{\sqrt{\mathrm{C}}}{4} \mathrm{f}_{1}, \dot{\mathrm{L}}_{3}=\frac{1}{8} \mathrm{f}_{2}-\frac{1}{2 \mathrm{C}_{0}} \mathrm{f}_{1} \\
& \dot{\mathrm{L}}_{2}=-\frac{1}{8} \mathrm{f}_{3}, \dot{\mathrm{L}}_{1}=\frac{\sqrt{\mathrm{C}}}{4} \mathrm{f}_{4}+\frac{1}{2 \mathrm{C}_{0}} \mathrm{f}_{3}
\end{aligned}
$$

Integrating we get,

$$
\begin{aligned}
& \dot{\mathrm{L}}_{1}=\left(-\frac{\tau \rho^{2}}{\mathrm{C}_{0}}+\frac{3 \mathrm{t}_{0}}{4 \mathrm{C}_{0}}\right) \mathrm{C}_{1}-\frac{\sqrt{\mathrm{C}}}{4} \mathrm{~b}_{4}+\frac{1}{2 \mathrm{C}_{0}} \mathrm{~b}_{3} \\
& \mathrm{~L}_{2}=\tau \mathrm{b}_{1}+\frac{\mathrm{t}_{0}}{8} \mathrm{C}_{1}-\frac{1}{8} \mathrm{~b}_{3} \\
& \mathrm{~L}_{3}=\left(-\frac{\rho^{2}}{4}+\frac{\rho_{(0)}^{2}}{4}\right) \mathrm{C}_{1}+\frac{1}{8} \mathrm{~b}_{2}-\frac{1}{2 \mathrm{C}_{0}} \mathrm{~b}_{1} \\
& \mathrm{~L}_{4}=\frac{\sqrt{\mathrm{C}}}{4} \mathrm{~b}_{1} \\
& \mathrm{~b}_{1}=\int_{0}^{\tau} 16 \mathrm{pqr} \mathrm{r}_{1}\left(\frac{1}{\mathrm{r}_{2}^{3}}-1\right) \mathrm{d} \tau \\
& \mathrm{b}_{2}=-\left(\frac{\partial \Omega}{\partial \mu}\right)+\left(\frac{\partial \Omega}{\partial \mu}\right) \tau=0, \mu=0 \\
& \mathrm{~b}_{3}=\int_{0}^{\tau}\left\{-8\left(\mathrm{p}^{4}-\mathrm{q}^{4}\right)\left(2+\frac{1}{\mathrm{r}_{2}^{3}}\right)+\frac{8 \mathrm{r}_{1}}{\mathrm{r}_{2}^{3}}\right\} \mathrm{d} \tau+16 \int_{0}^{\tau} \operatorname{tpqr}_{1}\left(\frac{1}{\mathrm{r}_{2}^{3}}-1\right) \mathrm{d} \tau+12 \mathrm{C}_{0}^{\prime} \int_{0}^{\tau} \mathrm{r}_{1}^{3} \mathrm{~d} \tau \\
& \mathrm{b}_{4}=\int_{0}^{\tau}\left[\left\{\tan \left(2 \sqrt{\mathrm{C} \tau}-\phi_{0}\right)-\frac{\mathrm{t}}{\mathrm{n}}\right\} \frac{\mathrm{db} \mathrm{b}_{1}}{\mathrm{~d} \tau}+2 / \mathrm{n} \tau \frac{\mathrm{db} \mathrm{b}_{2}}{\mathrm{~d} \tau}\right] \mathrm{d} \tau
\end{aligned}
$$

In general, solution of the system (19) 


$$
\mathrm{x}_{\mathrm{k}}^{(1)}=\sum_{\mathrm{i}=1}^{4}\left(\mathrm{~L}_{\mathrm{i}}+\mathrm{a}_{\mathrm{i}}\right) \mathrm{y}_{\mathrm{ki}},(\mathrm{k}=1, \ldots . .4)
$$

There will enter six constraints $h, C, a_{i}(i=1, \ldots \ldots 4)\left(a_{i}=\right.$ constants of integration $)$ which must be chosen in such a manner that the following relations are satisfied

$$
\mathrm{x}_{2}^{(1)}(0)=\mathrm{x}_{3}^{(1)}(0)=\left(\frac{\mathrm{d} \Omega}{\mathrm{d} \mu}\right)_{\mu=0}=\mathrm{x}_{4}^{(1)}\left(\mathrm{S}^{*}\right)=0, \mathrm{~S}^{*}=\frac{\pi \mathrm{k}}{4 \sqrt{\mathrm{C}}_{0}}, \text { if }(\mathrm{k}+\mathrm{m}) \text { is odd }(30)
$$

and $x_{2}^{(1)}(0)=x_{3}^{(1)}(0)=\left(\frac{d \Omega}{d \mu}\right)_{\mu=0}=x_{1}^{(1)}\left(S^{*}\right)=x_{4}^{(1)}\left(S^{*}\right)=0, S^{*}=\frac{\pi k}{4 \sqrt{C}}$, if $k+m$ is even $(31)$

From the relations

$\mathrm{x}_{2}^{(1)}(0)=\mathrm{x}_{3}^{(1)}(0)=0$

we find immediately that $\mathrm{a}_{1}=\mathrm{a}_{2}=0$

we shall write the equations (30) and (31) in the following manner

$$
\begin{aligned}
& x_{i}^{(1)}\left(s^{*}\right)=F^{*}{ }_{i 1} h_{1}+F^{*}{ }_{i 2} C_{1}+F^{*}{ }_{i 3} a_{3}+F^{*}{ }_{i 4} a_{4}+F^{*}{ }_{i}=0 \\
& x_{j}^{(1)}\left(s^{*}\right)=F^{*}{ }_{j 1} h_{1}+F^{*}{ }_{j 2} C_{1}+F^{*}{ }_{j 3} a_{3}+F^{*}{ }_{j 4} a_{4}+F^{*}{ }_{j}=0 \\
& \left(\frac{d \Omega}{d \mu}\right)_{\mu=0}=\frac{4}{C_{0}} C_{1}+\frac{16}{n} a_{3}+\left(\frac{d \Omega}{d \mu}\right)_{\tau=0}
\end{aligned}
$$

Where

$$
\begin{aligned}
& \mathrm{i}=1, \mathrm{j}=4, \mathrm{k}+\mathrm{m} \text { is odd } \\
& \mathrm{i}=2, \mathrm{j}=3, \mathrm{k}+\mathrm{m} \text { is even } \\
& \mathrm{F}_{\mathrm{k} 1}=\tau \mathrm{y}_{\mathrm{k} 2}, \mathrm{~F}_{\mathrm{k} 3}=\mathrm{y}_{\mathrm{k} 3}, \mathrm{~F}_{\mathrm{k} 4}=\mathrm{y}_{\mathrm{k} 4} \\
& \mathrm{~F}_{\mathrm{k} 2}=\left(\frac{\tau \rho^{2}}{\mathrm{C}_{0}}-\frac{3 \mathrm{t}_{0}}{4 \mathrm{C}_{0}}\right) \mathrm{y}_{\mathrm{k} 1}+\frac{\mathrm{t}_{0}}{8} \mathrm{y}_{\mathrm{k} 2}\left(\frac{\rho^{2}}{4}+\frac{\rho^{2}(0)}{4} \mathrm{y}_{\mathrm{k} 3}\right) \\
& \mathrm{F}_{\mathrm{k}}=\left(-\frac{\sqrt{\mathrm{C}}}{4} \mathrm{~b}_{4}+\frac{1}{2 \mathrm{C}_{0}} \mathrm{~b}_{3}\right) \mathrm{y}_{\mathrm{k} 1}-\frac{1}{8} \mathrm{~b}_{3} \mathrm{y}_{\mathrm{k} 2}+\left(\frac{1}{8} \mathrm{~b}_{2}+\frac{1}{2 \mathrm{C}_{0}} \mathrm{~b}_{1}\right) \mathrm{y}_{\mathrm{k} 3}+\frac{\sqrt{\mathrm{C}_{0}}}{4} \mathrm{~b}_{1} \mathrm{y}_{\mathrm{k} 4}
\end{aligned}
$$

where $\mathrm{h}_{1}$ are defined by the formula (28).

Thus we have three equations for the determination of four constants $\left(h_{1}, c_{1}, a_{3}, a_{4}\right)$. One of them is chosen arbitrary when $\mathrm{k}$ is even $\mathrm{m}$ is odd, $\phi_{0}=0, \omega= \pm \pi / 2$ one may require in order that in perturbed as well as in unperturbed motion the period in independent variable $t$ may coincide; then to these equations we shall supplement another.

$$
\mathrm{t}\left(\tau^{*}, \mu\right)-\pi \mathrm{m}=0, \tau^{*}=\frac{\pi \mathrm{k}}{4 \sqrt{\mathrm{C}}_{0}}
$$

where $t$ may be put in the form of the series in $\mu$. 


$$
\mathrm{t}=\mathrm{t}_{0}+\sum_{\mathrm{i}=1}^{\infty} \mathrm{t}_{\mathrm{i}} \mu^{\mathrm{i}}, \mathrm{t}_{\mathrm{i}}(0)=0
$$

Let us pass in the expression (6) to new independent variation

$$
\begin{aligned}
& \tau=\frac{\mathrm{S}^{*}}{\mathrm{~S}^{*}+\alpha} \mathrm{S} \\
& \frac{\mathrm{dt}}{\mathrm{d} \tau}=4 \frac{\mathrm{S}^{*}+\alpha}{\mathrm{S}^{*}}\left(\mathrm{x}_{1}^{2}+\mathrm{x}_{2}^{2}\right)
\end{aligned}
$$

And we shall substitute expression (35) in (36) then for the determination of $t_{1}$, we have the following equations.

$$
\frac{\mathrm{dt}}{\mathrm{d} \tau}=4\left(\mathrm{x}_{1}^{(0)^{2}}+\mathrm{x}_{2}^{(0)^{2}}\right) \mathrm{h}_{1}+8\left(\mathrm{x}_{1}^{(0)} \mathrm{x}_{1}^{(1)}+\mathrm{x}_{1}^{(0)} \mathrm{x}_{1}^{(1)}\right)
$$

Integrating we obtain

$$
\mathrm{t}_{1}=\mathrm{F}_{51} \mathrm{~h}_{1}+\mathrm{F}_{52} \mathrm{C}_{1}+\mathrm{F}_{53} \mathrm{a}_{3}+\mathrm{F}_{54} \mathrm{a}_{4}+\mathrm{F}_{5} .
$$

where

$$
\begin{aligned}
& \mathrm{F}_{51}=4\left(\mathrm{p}_{0}^{2}+\mathrm{q}_{0}^{2}\right) \\
& \mathrm{F}_{52}=\frac{\mathrm{t}_{0}\left(\mathrm{p}_{0}^{2}+\mathrm{q}_{0}^{2}\right)}{52}+\frac{\mathrm{t}_{0}}{\mathrm{C}_{0}}-\frac{4 \sqrt{\mathrm{C}} \sin 2 \sqrt{\mathrm{C}_{0}}}{\mathrm{C}_{0}}+\frac{4 \sin ^{2} 2 \sqrt{\mathrm{C}}}{3 \mathrm{C}_{0}} \\
& \mathrm{~F}_{53}=2 \mathrm{t}_{0}, \mathrm{~F}_{54}=\frac{8\left(\mathrm{p}_{0}^{2}+\mathrm{q}_{0}^{2}\right)}{\mathrm{n}}+\frac{2 \mathrm{t}_{0}}{\mathrm{n}} \\
& \mathrm{F}_{5}=\int_{0}^{\tau}\left(\mathrm{L}_{2}+\frac{2}{\mathrm{n}} \mathrm{L}_{4}\right)(\mathrm{p} \dot{\mathrm{p}}+\mathrm{q} \dot{\mathrm{q}}) \mathrm{L}_{3}\left(\mathrm{p}^{2}+\mathrm{q}^{2}\right) \mathrm{d} \tau
\end{aligned}
$$

Then $t_{0}$ the equation (32) when $\mathrm{k}$ is even, $\mathrm{m}$ is odd, $\phi=0, \omega= \pm \pi / 2$, one may add also another equation.

$$
\mathrm{t}_{1}\left(\tau^{*}\right)=\mathrm{F}_{51}^{*} \mathrm{~h}_{1}+\mathrm{F}_{52}^{*} \mathrm{C}_{1}+\mathrm{F}_{53}^{*} \mathrm{a}_{3}+\mathrm{F}_{54}^{*} \mathrm{a}_{4}+\mathrm{F}_{5}^{*}=0
$$

This is the required equation for calculating the first order perturbation in centrifugal forces.

\section{Acknowledgements}

We are thankful to Prof. Dr. Mrityunjay Jha, Prof. and Head, University Department of Mathematics, T.M.B., University; Bhagalpur, India for giving us encouragement and his valuable suggestions.

\section{References}

1. H. Poincare, Les Mathod nouvels dela mecanique cilest., Paris, 1892.

2. I.V. Kurcheeva, Bulletin, Inst. Of Theo. Astro. (ITA) (1968)149.

3. A. Ahmad, Ph.D. thesis, Bhagalpur University, Bhagalpur, 1981.

4. A. Ahmad, and A.A. Khan, JBMS, 10(86) 199-128.

5. K.B. Bhatnagar, and P.P. Hallen, Celestial Mechanics 18 (1978) 105.

6. T. Levi-Civita, Periodic orbits, Washington, 1906.

7. G.A. Krasinski, Bulletin, Inst. of Theo. Astro. (ITA) 9 (1963) 105. 